\title{
Global existence and uniqueness of the solution to a nonlinear parabolic equation
}

\section{Alexander G. Ramm}

\author{
Mathematics Department, Kansas State University, \\ Manhattan, KS 66506-2602, USA
}

\begin{abstract}
Consider the equation

$$
u^{\prime}(t)-\Delta u+|u|^{\rho} u=0, \quad u(0)=u_{0}(x),(1),
$$

where $u^{\prime}:=\frac{d u}{d t}, \rho=$ const $>0, x \in \mathbb{R}^{3}, t>0$.

Assume that $u_{0}$ is a smooth and decaying function,

$$
\left\|u_{0}\right\|=\sup _{x \in \mathbb{R}^{3}, t \in \mathbb{R}_{+}}|u(x, t)| .
$$

It is proved that problem (1) has a unique global solution and this solution satisfies the following estimate$$
\|u(x, t)\|<c
$$

where $c>0$ does not depend on $x, t$.

Mathematics Subject Classification: MSC 2010, $35 \mathrm{~K} 55$.

Keywords: nonlinear parabolic equations; global solutions.

\section{Introduction}

Let

$$
u^{\prime}-\Delta u+|u|^{\rho} u=0, \quad u(0)=u_{0} ; \quad u^{\prime}:=\frac{d u}{d t},
$$

where $\rho>0, t \in \mathbb{R}_{+}=[0, \infty), x \in \mathbb{R}^{3}, X$ is a Banach space of real-valued functions with the norm $\|u(x, t)\|:=\sup _{x \in \mathbb{R}^{3}, t \in R_{+}}|u(x, t)|$. We assume that

$$
\|u\| \leq c
$$

Corresponding author: Email: ramm@ksu.edu 
We say that $u$ is a global solution to (1) if $u$ exists $\forall t \geq 0$.

Our result is formulated in Theorem 1. Our method is simple and differs from the published results, see [1, [2] and references there.

The novel points in this work are:

a) There is no restriction on the upper bound of $\rho$.

In [1], (Section 1.1) a nonlinear hyperbolic equation with the same nonlinearity is studied in a bounded domain, uniqueness of the solution is proved only for $\rho \leq 2 /(n-2)$, and existence is proved by a different method. The contraction mapping theorem is not used.

In [2] the quasi-linear problems for parabolic equations are studied in Chapter 5 in a bounded domain and under the assumptions different from ours. There are many papers and books on non-linear problems for parabolic equations (see the bibliography in [1], [2].

b) Existence of the global solution is proved.

c) Method of the proof differs from the methods in the cited literature.

Our result is formulated in Theorem 1:

Theorem 1. Problem (1) has a unique global solution in $X$ for any $u_{0} \in X$.

\section{Proofs}

Let $g(x, t)=\frac{e^{-|x|^{2}}}{(4 \pi t)^{3 / 2}}$. If $u$ solves (1) then

$$
\begin{gathered}
u(t)=-\int_{0}^{t} d \tau \int g(x-y, t-\tau)|u|^{\rho} u d y+ \\
\int g(x-y, t) u_{0}(y) d y:=A(u)+F:=Q(u),
\end{gathered}
$$

where $\int:=\int_{\mathbb{R}^{3}}$. Let $X$ be the Banach space of continuous in $\mathbb{R}^{3} \times R_{+}$functions, $\mathbb{R}_{+}:=[0, \infty),\|u\|:=\max _{x \in \mathbb{R}^{3}, t \in[0, T]}|u(x, t)|$. If $\|u\| \leq R$ then $\|A(u)\| \leq$ $T R^{\rho+1}$, where the identity $\int g(x-y, t-\tau) d y=1$ was used. From (3) one gets

$$
\|u\| \leq T\|u\|^{\rho+1}+\|F\| .
$$

Thus, $Q$ maps the ball $B(R)=\{u:\|u\| \leq R\}$ into itself if $T$ is such that

$$
T R^{\rho+1}+\|F\| \leq R
$$

The $Q$ is a contraction on $B(R)$ if

$$
\|Q(u)-Q(v)\| \leq T(\rho+1) R^{\rho}\|u-v\| \leq q\|u-v\|, \quad 0<q<1 .
$$

Thus, if

$$
T(\rho+1) R^{\rho} \leq q<1
$$


then $Q$ is a contraction in $B(R)$ in the Banach space $X_{T}$ with the norm $\|\cdot\|$, $t \in[0, T]$. We use the same notations for the norms in $X_{T}$ and in $X_{\infty}$.

We have proved that

For $T$ satisfying (5) - (6) there exists and is unique the solution to (1), and this solution can be obtained from (3) by iterations.

The problem now is:

Does this solution exist and is unique on $R_{+}$?

From our proof it follows that if the solution exists and is unique in $X_{T}$, then the solution exists and is unique in $X_{T_{1}}$ for some $T_{1}>T$.

To prove that the solution $u(x, t)$ to (1) exists on $\mathbb{R}_{+}$, assume the contrary: this solution does not exist on any interval $\left[0, T_{1}\right), T_{1}>T$, where $T$ is the maximal interval of the existence of the continuous solution. Then $\lim _{t \rightarrow T-0} u(x, t)=\infty$, because otherwise there is a sequence $t_{n} \rightarrow T-0$ such that $u\left(x, t_{n}\right) \rightarrow u(x, T)$ and one may construct the solution defined on [T, $\left.T_{1}\right]$, $T_{1}>T$, by using the local existence and uniqueness of the solution to (1) with the initial value $u(x, T)$ for $t \in\left[T, T_{1}\right]$. This contradicts the assumption that $T$ is the maximal interval of the existence of the continuous solution $u$.

Thus, if $T<\infty$ then one has $\lim _{t \rightarrow T-0} u(x, t)=\infty$. Let us prove that this also leads to a contradiction. Then we have to conclude that $T=\infty$ and Theorem 1 is proved.

We need some estimates. Multiply (1) by $u$, integrate over $\mathbb{R}^{3}$ with respect to $x$, and then integrate by parts the second term. The result is:

$$
0.5 \frac{d N(u)}{d t}+N(\operatorname{grad} u)+\int|u|^{\rho+2} d y=0,
$$

where $N(u):=\int u^{2} d y$. Integrate (7) with respect to time over $[0, T]$ and get

$$
0.5 N(u(T))+\int_{0}^{T}\left(N(\operatorname{grad} u)+\int|u|^{\rho+2} d y\right) d \tau=0.5 N(u(0)) .
$$

Therefore,

$$
N(u(t)) \leq c, \forall t \in[0, T], \quad \int_{0}^{T} N(\operatorname{grad} u) d \tau \leq c, \quad \int_{0}^{T} d \tau \int|u|^{\rho+2} d y \leq c,
$$

where $c=0.5 N\left(u_{0}\right)$.

Lemma 1. From (9) and (3) it follows that

$$
\|u(x, t)\|<\infty \quad \forall t \in[0, T] .
$$

If (10) is proved then $T$ is not the maximal interval of the existence of the solution to (1). This contradiction proves Theorem 1. 
Proof of Lemma 1. One uses the Hölder inequality twice and gets

$$
\begin{array}{r}
\int_{0}^{T} d \tau \int g(x-y, t-\tau)|u|^{\rho+1} d y \leq \\
\left(\int_{0}^{T} d \tau \int|u|^{\rho+2} d y\right)^{(\rho+1) /(\rho+2)}\left(\int_{0}^{T} d \tau \int g^{\rho+2} d y\right)^{1 /(\rho+2)} \leq \\
\left(\int_{0}^{T} d \tau \int|u|^{\rho+2} d y\right)^{(\rho+1) /(\rho+2)}\left(\int_{0}^{T} d \tau \int g^{\rho+2} d y\right)^{1 /(\rho+2)}
\end{array}
$$

By the last inequality (9) it follows that $\int_{0}^{T} d \tau \int|u|^{\rho+2} d y<c \forall T>0$, where $c>0$ is a constant independent of $T$. The last integral in 11 is also bounded independently of $T$. It can be calculated analytically.

Thus, inequalities (11), (9) and equation (3) imply (10).

Lemma 1 is proved.

Therefore Theorem 1 is proved.

The ideas related to the ones used in this paper were developed and used in $[3]-[5]$.

\section{References}

[1] Lions, J., Quelques methods de resolution des problemes aux limites non lineaires, Dunod, Paris, 1969.

[2] Ladyzhenskaya, O., et al.,Linear and quasilinear equations of parabolic type, Transl. of math. monogr., vol. 23, Amer. Math. Soc., Providence RI, 1968.

[3] Ramm, A. G., Stability of the solutions to evolution problems, Mathematics, 1, (2013), 46-64.

doi:10.3390/math1020046

Open access Journal:

http://www.mdpi.com/journal/mathematics

[4] Ramm, A. G., Large-time behavior of solutions to evolution equations, Handbook of Applications of Chaos Theory, Chapman and Hall/CRC, 2016, pp. 183-200 (ed. C.Skiadas).

[5] Ramm, A. G., Hoang, N. S., Dynamical Systems Method and Applications. Theoretical Developments and Numerical Examples. Wiley, Hoboken, 2012. 